\title{
On the dynamic behavior of the Zener model with nonlinear stiffness for harmonic vibration isolation
}

\author{
Lucas de Haro Silva ${ }^{a}$, Paulo J. Paupitz Gonçalves ${ }^{\mathrm{a}, *}$, David Wagg ${ }^{\mathrm{b}}$ \\ a School of Engineering of Bauru, São Paulo State University - UNESP, Brazil \\ ${ }^{\mathrm{b}}$ Department of Mechanical Engineering, University of Sheffield, United Kingdom
}

\section{A R T I C L E I N F O}

\section{Article history:}

Received 15 November 2017

Received in revised form 12 March 2018

Accepted 21 April 2018

\section{Keywords:}

Vibration isolation

Nonlinear

Zener model

\begin{abstract}
A B S T R A C T
In this paper, the dynamic behaviour of a mechanical isolator known as the Zener model is considered. This model consists of a mass supported by a spring that is in parallel with two further elements in series; a damper and a second spring. Previous work had already demonstrated that this system has benefits for the transmissibility above the resonance frequency, when compared with the traditional spring-damper isolator. In this work we study the influence of replacing the main spring with a nonlinear cubic spring. In fact, we show that use of a cubic stiffness can provide improvement in the transmissibility for frequencies around and above the resonance if the stiffness is of the softening type. Results are based on the analytical development of the equation of motion using the harmonic balance method.
\end{abstract}

(c) 2018 Elsevier Ltd. All rights reserved.

\section{Introduction}

In linear vibration theory, when considering discrete elements subjected to base excitation, such as the single degree-offreedom (SDOF) spring mass damper system, there is the concept of transmissibility (absolute and relative). The absolute displacement transmissibility is defined as the ratio of the displacement of the mass to the displacement of the base.

Vibration isolation occurs when the transmissibility is less than one (displacement of the mass is smaller than the base displacement). For a SDOF spring mass damper system this occurs for frequencies higher than $\omega_{0} \sqrt{2}$, where $\omega_{0}$ is the resonance frequency of the undamped system. Therefore, in order to maximize the isolation region, it would make sense to have the resonance frequency as low as possible but this typically results in low stiffness or large mass which cause undesirably large static deformations.

Increasing the viscous damping coefficient, $c$, reduces the transmissibility near the resonance, but degrades the high frequency response. It is well known that the high frequency transmissibility of a harmonically forced spring-viscous damper isolator reduces at the rate $2 \xi_{0} / \Omega$ where $\xi_{0}=c / 2 m \omega_{0}$ is the damping ratio and $\Omega=\omega / \omega_{0}$ is the frequency ratio, where $m$ is the mass and $\omega$ the base excitation frequency.

Therefore, the requirements for the design of a new vibration isolation system are naturally focused on defining a system with low transmissibility at resonance without degrading its high frequency performance. An optimal solution for this type of isolator cannot be achieved using linear theory, hence, exploring the non-linear behaviour of mechanical components is a potential solution for this problem.

\footnotetext{
* Corresponding author.

E-mail address: paulo.jpg@feb.unesp.br (P.J. Paupitz Gonçalves).
} 
For instance, Ibrahim [1] has presented a comprehensive literature review of nonlinear passive vibration isolators prior to the year 2008. Since then, there were many other research papers discussing the use of nonlinear elements for the benefit of vibration isolation. In the work of Kovacic et al. [2], the authors proposed the study of a combination of linear and inclined pre-stressed springs which were both geometrically and physically nonlinear. An optimal combination of the system parameters is found that maximizes the displacement from the equilibrium position when the prescribed stiffness is equal to that of the vertical spring alone. Carrella et al. [3] have also proposed the use of nonlinear elements to achieve high static and low dynamic stiffness (HSLDS). Similar analysis was presented by Carrella et al. [4] with the use of magnets to provide nonlinear behavior of the isolator system and by Shaw et al. [5] where the authors present nondimensional analysis that shows the response of HSLDS mounts subject to harmonic excitation, relative to an equivalent linear system demonstrating the effects of nonlinearity.

The work Guo et al. [6] studied a nonlinear viscous damper used as an energy dissipating device for the force and displacement transmissibility of a single degree-of-freedom system. Analytical algorithms are derived using the RitzGalerkin method. Peng et al. [7] study the influence of cubic nonlinear damping in a vibration isolator using the harmonic balance method. They claim that cubic nonlinear damping has no effect on the transmissibility in the non-resonance region. Similar damping characteristics were also investigated by Lang et al. [8]. Tang et al. [9] considered the free vibration of single degree of a freedom oscillator with geometrically nonlinear damping, such that the spring and the damper are connected to the mass so that they are orthogonal, and the vibration is in the direction of the spring. It is shown that, provided the displacement is small, this system behaves in a similar way to the conventional SDOF system with cubic damping, in which the spring and the damper are connected so they act in the same direction.

Peng et al. [10] studied the use of antisymmetric damping of a single degree-of-freedom system. They have found that this type of isolator has almost no effect at low and high frequency ranges, but it can significantly improve the transmissibility over the region close to the resonance frequency. An isolator with a damping factor that is proportional to both velocity and position was studied by Xiao et al. [11] which also shows improvement of the transmissibility when compared to the traditional linear spring-damper isolator. Other authors such as Milovanovic et al. [12], Kovacic [13] and Ho et al. [14] have developed studies on similar subjects, considering both nonlinear damping and stiffness elements.

Sun and Jing $[15,16]$ have design a scissor-like (X-shaped) multiple direction vibration isolator using the concept of to achieve Quasi-Zero-Stiffness. The X-shaped isolator provided geometrically nonlinear effects on both linear spring and damper. The authors claim that their design provides lower resonant frequency and peak values, stable equilibrium without jumping phenomenon, and robustness to mass changes.

The Zener model is often referred to as a standard linear solid model used to represent the viscoelastic behavior of materials. Other similar models, such as the Maxwell and the Kelvin-Voigt model are usually insufficient to represent materials that exhibit viscoelastic rheology.

The linear behavior of the Zener model has been described by Ruzicka [17,18] and Brennan et al. [19], however to the authors knowledge there are no papers discussing the influence of nonlinear elements in this model.

It is already known that this isolator arrangement presents benefits in frequencies above the resonance, since the high frequency transmissibility asymptote of the linear Zener isolator is defined by $|T|=(1+N)\left(\omega_{0} / \omega\right)^{2}\left(\right.$ where $\omega_{0}$ is the resonance frequency of the linear spring mass system). Therefore, in this paper we study the effect of adding a nonlinear cubic stiffness to the transmissibility of the system.

The main finding of the paper is that adding a nonlinear stiffness of the softening type improves the transmissibility at high frequencies without significantly affecting the transmissibility around the resonance region.

The structure of this work is presented as follows; Section 2 presents a review of the linear Zener model to be used for comparison; Section 3 presents the modelling procedure of the nonlinear system; Section 4 describes the methodology to obtain the approximated transmissibility response using the harmonic balance method; Section 5 shows a study of the stability of the periodic response describing the frequency ranges where jump phenomena can occur. In Section 7 numerical integration is used to verify the results obtained by the harmonic balance method. Finally, Section 8 presents the main conclusions of this paper.

\section{Model definition}

The system under study is shown in Fig. 1 representing a mass supported by a main spring $k$ and a series combination of a secondary spring $k_{2}$ and a linear viscous damper with coefficient $c$. The system is subject to base motion with amplitude $x_{0}$, the mass displacement is defined by $x$ and the variable $x_{1}$ is related to the degree-of-freedom where the viscous damper and the secondary spring are connected.

The equations of motion of the system shown in Fig. 1 are expressed by the dynamic equilibrium on the mass and the force equilibrium in degree-of-freedom $\left(x_{1}\right)[17,18]$

$$
\begin{aligned}
& m \ddot{x}=-k\left(x-x_{0}\right)-c\left(\dot{x}-\dot{x}_{1}\right), \\
& c\left(\dot{x}-\dot{x}_{1}\right)=k_{2}\left(x_{1}-x_{0}\right) .
\end{aligned}
$$




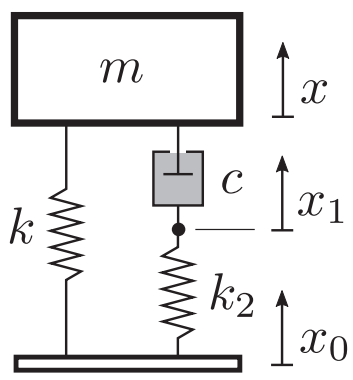

Fig. 1. Representation of Zener model subject to base motion.

Assuming that $k_{2}=N k$, where $N$ is a positive real number, it is more convenient to write the two differential equations (Eqs. (1) and (2)) as one higher order Ordinary Differential Equation (ODE). This can be done by substituting the term on the right hand side of Eq. (2) into Eq. (1), and differentiating once in respect to time

$$
\dot{x}_{1}=-\frac{1}{N k}\left(m \ddot{x}+k\left(\dot{x}-\dot{x}_{0}\right)\right)+\dot{x}_{0} .
$$

The variable $\dot{x}_{1}$ is then replaced in Eq. (1) to provide a third order ODE. defined as

$$
\left(\frac{2 \xi_{0}}{\omega_{0} N}\right) \ddot{x}+\ddot{x}+2 \xi_{0} \omega_{0}\left(\frac{N+1}{N}\right) \dot{x}+\omega_{0}^{2} x=2 \xi_{0} \omega_{0}\left(\frac{N+1}{N}\right) \dot{x}_{0}+\omega_{0}^{2} x_{0} .
$$

For convenience the system parameters where simplified to $\omega_{0}=\sqrt{k / m}, \xi_{0}=c / 2 m \omega_{0}$. (Note that these are the natural frequency and damping ratio of a mass-spring-damper system and not the natural frequency and damping ratio the system under study).

Considering the special case where the base displacement is a harmonic function of the type $x_{0}=X_{0} e^{j \omega t}$, with $j^{2}=-1$, and $\omega$ is the frequency of the external excitation. Assuming that the response of the system is also a harmonic function of the type $x=X \mathrm{e}^{\mathrm{j}(\omega t+\beta)}$, where $\beta$ is the phase angle between the base displacement $x_{0}$ and the mass displacement $x$, then in the steady state regime, the transmissibility of the system can be written as

$$
\frac{X}{X_{0}}=\frac{j \Omega(N+1)+\alpha}{\alpha\left(1-\Omega^{2}\right)+j \Omega\left(N+1-\Omega^{2}\right)},
$$

where $\alpha=N / 2 \xi_{0}$ and $\Omega=\omega / \omega_{n}$ is the frequency ratio. The magnitude of the transmissibility is then defined as

$$
|T|=\left(\frac{\Omega^{2}(N+1)^{2}+\alpha^{2}}{\alpha^{2}\left(1-\Omega^{2}\right)^{2}+\Omega^{2}\left(N+1-\Omega^{2}\right)^{2}}\right)^{1 / 2},
$$

and the phase angle $\beta$ is given by

$$
\beta=\operatorname{atan}\left(\frac{-\alpha \Omega^{3} N}{-(N+1) \Omega^{4}+\left(-\alpha^{2}+(N+1)^{2}\right) \Omega^{2}+\alpha^{2}}\right) .
$$

Brennan et al. [19] have demonstrated that critical damping only occurs if there is a minimum value of $N$ which must be greater than 8 , otherwise the secondary spring is too soft to permit the damper to operate in the critical damping regime. If $N$ is small, the system will behave as an underdamped system. If the damping ratio is to high, it will "block" the damper and the system will behave as an undamped system, with resonance frequency $\Omega=\sqrt{1+N}$. The factors that control the resonance peak are the terms in the denominator of Eq. (6), which depend on the value of $\alpha$. This can be verified in the plots of Fig. 2(a) and (b) which illustrate the influence of the damping factor $\xi_{0}$ in the terms defining the denominator of Eq. (6).

The general behavior of the transmissibility as a function of the frequency and the damping factor is shown in the surface plot of Fig. 3. It can be seen that there is a shift in the resonance frequency as the damping factor increases. This is because there is a change in the roots of the polynomial defined in the denominator of Eq. (5), as $\xi_{0}$ is changed [19]. Increasing $\xi_{0}$ from small to large values, the transmissibility passes through a minimum and increases again. According to [18,19], the minimum is defined by the optimal damping value as

$$
\xi_{o p t}=\frac{N \sqrt{2(N+2)}}{4(N+1)} .
$$




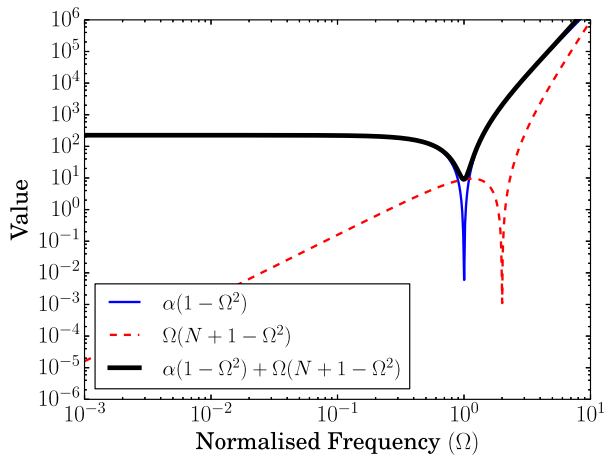

(a)

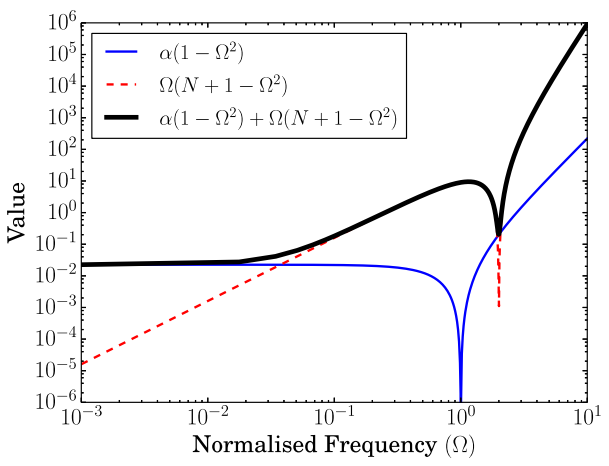

(b)

Fig. 2. Contribution of each term in the denominator of Eq. (6) for $N=3$. Case (a) $\xi_{0}=0.1$ and case (b) $\xi_{0}=4.0$.

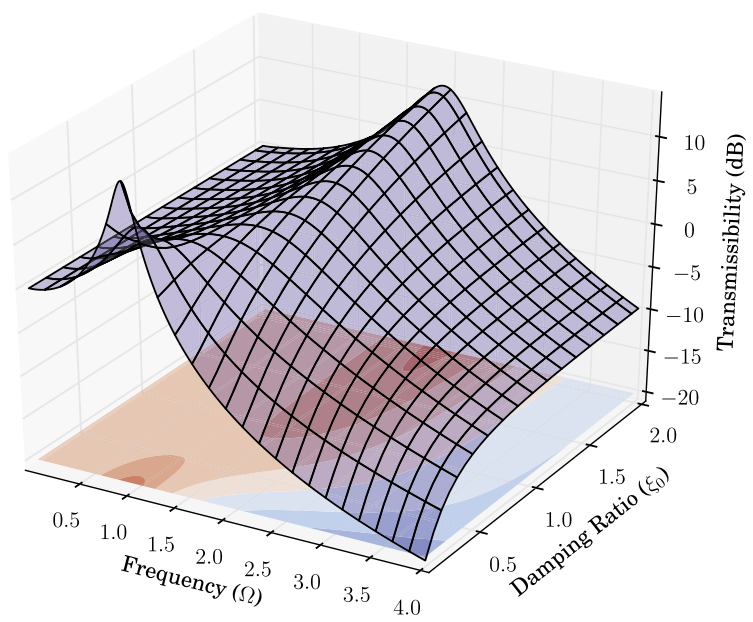

Fig. 3. Surface plot for the base transmissibility as a function the frequency and the damping ratio $\xi_{0},(N=3)$.

The linear spring-mass isolator provides isolation $(|T|<1)$ for frequencies above $\Omega=\sqrt{2}$. In the case of the Zener isolator, this frequency is given by

$$
\Omega_{\text {isolation }}=\frac{1}{\sqrt{2}}\left(2(N+1)-\alpha^{2}+\sqrt{\left(\alpha^{2}+2\right)^{2}+4 N\left(N+2-\alpha^{2}\right)}\right)^{1 / 2} .
$$

The magnitude of $\Omega_{\text {isolation }}$ varies between $\Omega_{l}=\sqrt{2}$ and $\Omega_{h}=\sqrt{2(N+1)}$ according to the values of $\xi_{0}$ and $N$, therefore the frequency of isolation in the linear Zener isolator is always greater than the spring-damper isolator. The value of magnitude of the transmissibility at high frequency can be found by keeping only the high order terms of $\Omega$ in both numerator and denominator of Eq. (6). This provides a high frequency asymptote defined by

$$
|T|=\frac{1+N}{\Omega^{2}} .
$$

The transmissibility has a high frequency behavior that does not depend on the system damping, but it is affected by the system mass and the stiffness of the two springs.

\section{Nonlinear cubic stiffness}

As observed in the last Section, the use of the Zener configuration of springs and dampers provides interesting isolation behavior for high frequency. This performance can be improved if low stiffness springs are used. For this reason, it is proposed to use a nonlinear stiffness of cubic type. The main spring $k$ is changed to an equivalent spring $k^{*}=k+k_{0}\left(x-x_{0}\right)^{2}$ and the spring force is defined by $f_{k^{*}}=k^{*}\left(x-x_{0}\right)$. The equation of motion is obtained according to the procedure presented 
in the last Section, which provides a third order nonlinear ODE, but this time in terms of the relative displacement $z=x-x_{0}$ and $z_{1}=x_{1}-x_{0}$,

$$
\begin{aligned}
& m \ddot{z}=-k z-k_{0} z^{3}-c\left(\dot{z}-\dot{z}_{1}\right)-m \ddot{x}_{0}, \\
& c\left(\dot{z}-\dot{z}_{1}\right)=N k z_{1} .
\end{aligned}
$$

By replacing the term on the right hand side of Eq. (12) in Eq. (11) and differentiating with respect to time, it is possible to obtain the expression,

$$
\dot{z}_{1}=-\frac{1}{N k}\left(m \ddot{z}+k \dot{z}+3 k_{0} z^{2} \dot{z}+m \ddot{x}_{0}\right)
$$

replacing the expression for $\dot{z}_{1}$ back in Eq. (11), the third order nonlinear ODE is obtained

$$
\frac{2 \xi_{0}}{N \omega_{0}} \stackrel{\cdots}{z}+\ddot{z}+2 \xi_{0} \omega_{0} \frac{(N+1)}{N} \dot{z}+\omega_{0}^{2} z+\gamma \omega_{0}^{2} z^{3}+\frac{6 \xi_{0}}{N} \gamma \omega_{0} z^{2} \dot{z}=-\ddot{x}_{0}-\frac{2 \xi_{0}}{N \omega_{0}} x_{0},
$$

where the parameters $c=2 \xi_{0} m \omega_{0}, \xi_{0}=2 m \omega_{0}$ and $\omega_{0}=\sqrt{k / m}$ were used. When damping ratio is vary small, (14) is approximated to

$$
\ddot{z}+\omega_{0}^{2} z+\gamma \omega_{0}^{2} z^{3}=-\ddot{x}_{0}, \quad \text { for small } \xi_{0},
$$

which is simple the equation of single harmonic oscillator with cubic stiffness. When the damping ratio is large, and keeping only the terms multiplied by $\xi_{0}$, Eq. (14) reduces to

$$
\ddot{z}+\omega_{0}^{2}(N+1) \dot{z}+3 \gamma \omega_{0}^{2} z^{2} \dot{x}=-x_{0}, \quad \text { for large } \xi_{0},
$$

which is equivalent to

$$
\ddot{z}+\omega_{0}^{2}(N+1) z+\gamma \omega_{0}^{2} z^{3}=-\ddot{x}_{0}, \quad \text { for large } \xi_{0} .
$$

Eqs. (15) and (17) represent the approximation for low and high damping for the Zener isolator with the influence of nonlinear stiffness.

\subsection{Reduction of dimensional parameters}

Introducing the nondimensional parameters

$$
\tau=\omega_{0} t, \quad \omega_{0} z^{\prime}=\dot{z}, \quad \gamma=k_{0} / k
$$

and assuming harmonic excitation from the base of the form $x_{0}=X_{0} \cos (\omega t)$, which when combined with the trigonometric terms of the right hand side of Eq. (14), gives

$$
z^{\prime \prime \prime}+\alpha z^{\prime \prime}+\left(N+1+3 \gamma z^{2}\right) z^{\prime}+\alpha\left(1+\gamma z^{2}\right) z=A \cos (\Omega \tau+\phi),
$$

where the prime denotes the derivative in respect to the nondimensional time variable $\tau$ and the base displacement amplitude is given by

$$
A=X_{0} \Omega^{2}\left(\alpha^{2}+\Omega^{2}\right)^{1 / 2},
$$

and the phase angle

$$
\phi=\operatorname{atan}(\Omega / \alpha) \text {. }
$$

\section{Approximated solution using the harmonic balance method}

To obtain an approximated solution for the nonlinear ODE, the method of harmonic balance is used. The solution of Eq. (18) is assumed to be of the form

$$
z=Z \cos (\Omega \tau+\phi+\theta)=Z \cos (\Psi), \quad \text { with } \Psi=\Omega \tau+\phi+\theta .
$$

where $\theta$ is the phase angle between the base excitation displacement given by right hand side of Eq. (18) and the relative displacement $z$ Applying this solution in Eq. (18) and neglecting terms with higher order harmonics $(\cos (3 \Psi), \sin (3 \Psi))$, the equation of motion is rewritten in the frequency domain as

$$
\alpha Z\left(1-\Omega^{2}+\frac{3}{4} \gamma Z^{2}\right) \cos (\Psi)-\Omega Z\left(N+1-\Omega^{2}+\frac{3}{4} \gamma Z^{2}\right) \sin (\Psi)=A \cos (\Psi-\theta) .
$$

Changing the right hand side cosine argument to match the terms on the left hand side, gives 


$$
\alpha Z\left(1-\Omega^{2}+\frac{3}{4} \gamma Z^{2}\right) \cos (\Psi)-\Omega Z\left(N+1-\Omega^{2}+\frac{3}{4} \gamma Z^{2}\right) \sin (\Psi)=A[\cos (\Psi) \cos (\theta)+\sin (\Psi) \sin (\theta)] .
$$

Equating the cosine and sine terms in both sides of the equation leads to

$$
\begin{aligned}
& \alpha Z\left(1-\Omega^{2}+\frac{3}{4} \gamma Z^{2}\right)=A \cos (\theta), \\
& -\Omega Z\left(N+1-\Omega^{2}+\frac{3}{4} \gamma Z^{2}\right)=A \sin (\theta) .
\end{aligned}
$$

For the free vibration case $(A=0)$, these equations provide a relationship between the oscillatory frequency as a function of the displacement amplitude, known as backbone curves. There are situations which depend on the amount of damping as has been seen for the linear case. These frequencies are shown in the results of Fig. 4 considering different two values of $\gamma$. The blue curves with resonance starting at $\Omega=1$ are calculated from Eq. (21) and the red curves starting at $\Omega=\sqrt{N+1}$ are calculated from Eq. (22). For the cases of negative $\gamma$, the maximum values of amplitude are defined at $\Omega=0$. For the first resonance frequency it is defined by $Z=\sqrt{\frac{4}{3 \gamma}}$ and for the second resonance frequency it is defined by $Z=\sqrt{\frac{4(N+1)}{3 \gamma}}$.

For the base excitation case, the relative transmissibility $Z$ can be obtained by combining Eqs. (21) and (22) to give an amplitude dependent frequency response function

$$
\left(\frac{Z}{X_{0}}\right)^{2}=\frac{\Omega^{4}\left(\alpha^{2}+\Omega^{2}\right)}{\alpha^{2}\left(1-\Omega^{2}+\frac{3}{4} \gamma Z^{2}\right)^{2}+\Omega^{2}\left(N+1-\Omega^{2}+\frac{3}{4} \gamma Z^{2}\right)^{2}} .
$$

Or the transmissibility can be written in terms of polynomial equation in $Z$

$$
a Z^{6}+b Z^{4}+c Z^{2}+d=0
$$

or a polynomial equation in terms of $\Omega$

$$
a_{1} \Omega^{6}+b_{1} \Omega^{4}+c_{1} \Omega^{2}+d_{1}=0 .
$$

Eqs. (24) and (25) are a closed form relation between the amplitude $Z$ and the excitation frequency $\Omega$ (The polynomial terms $a, b, c, d$ are shown in the appendix). Both equations when solved permit the estimation of frequency response as a function of the system parameters $N, \xi_{0}, \gamma$ and the input displacement $X_{0}$. Since the system is nonlinear, the behavior of the frequency response also changes with the amplitude. The results in Fig. 5 illustrate the behavior of positive and negative values $\gamma$ for two different values of damping ratio $\xi_{0}$. The results are compared with the linear case (thin black line). The frequency range around resonance is as expected, with the peak bending to the left for the softening $(\gamma<0)$ case and bending to right with the hardening case $(\gamma>0)$.

The phase angle $(\theta)$ between the base excitation and the relative transmissibility is obtained by the ratio of Eqs. (22) and (21), which gives

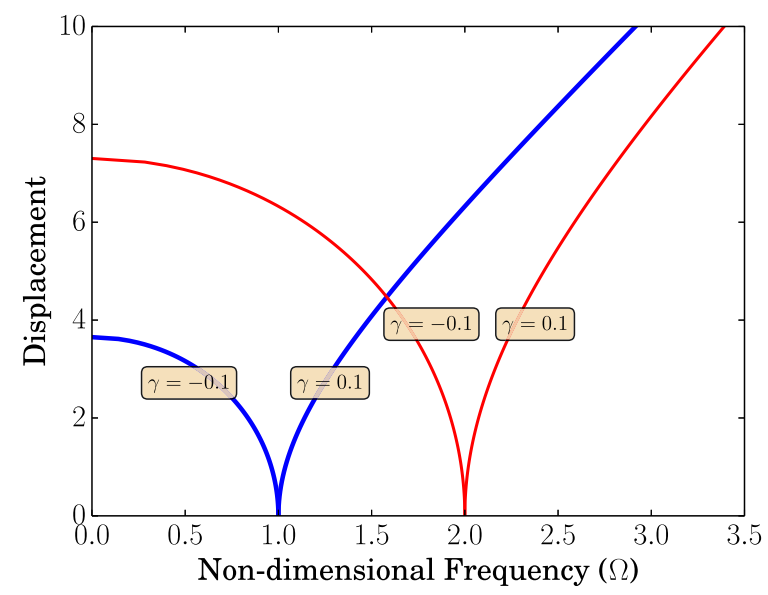

Fig. 4. Backbone curves (oscillation frequency as a function of the displacement amplitude. Blue thick lines are for the first resonance frequency $(\Omega=1)$ and thin red line are the second resonance frequency $(\Omega=\sqrt{1+N}$ ), with $N=3$ ). (For interpretation of the references to color in this figure legend, the reader is referred to the web version of this article.) 


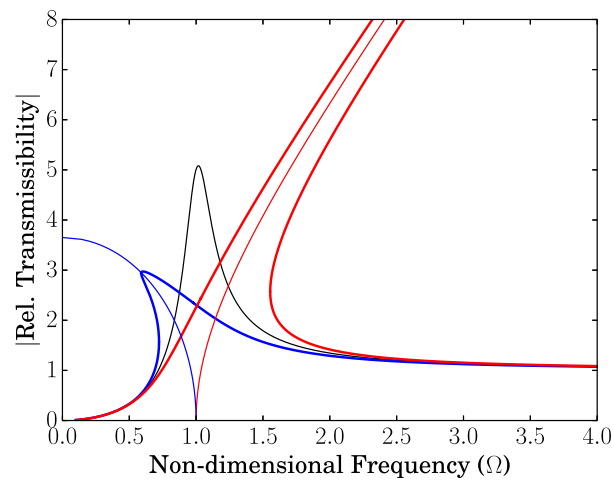

(a)

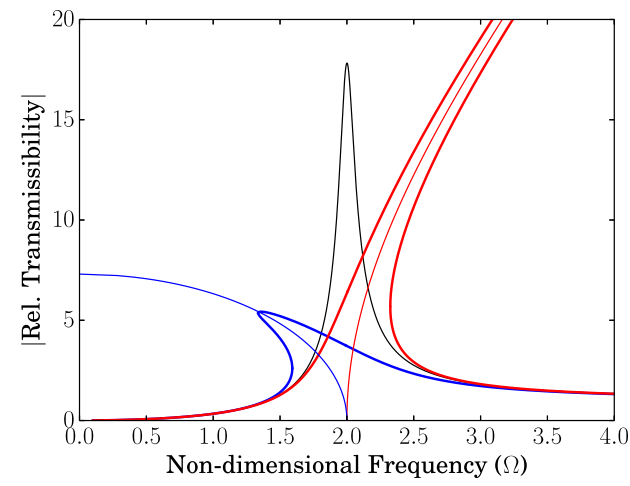

(b)

Fig. 5. Comparison of Relative Transmissibility for $N=3$. (a) Damping ratio $\xi_{0}=0.1$ with $\gamma=-0.1$ (blue curve bending to the left) and $\gamma=0.02$ (red curve bending to the right) (b) damping ratio $\xi_{0}=10.0$ with $\gamma=-0.1$ (blue curve bent to the left) and $\gamma=0.02$ (red curve bent to the right). (For interpretation of the references to color in this figure legend, the reader is referred to the web version of this article.)

$$
\theta=\operatorname{atan}\left(-\frac{\Omega}{\alpha}\left(\frac{N}{1-\Omega^{2}+3 / 4 \gamma Z^{2}}+1\right)\right) .
$$

\subsection{Absolute transmissibility}

Generally it is more informative to analyze the results in terms of absolute instead of the relative transmissibility. Note that the absolute displacement can be written as $x=z+x_{0}$, and that

$$
x=X \cos (\Omega \tau+\beta), \quad z=Z \cos (\Omega \tau+\theta+\phi) \quad \text { and } \quad x_{0}=X_{0} \cos (\Omega \tau) .
$$

The absolute displacement can be computed as

$$
X=\sqrt{Z^{2}+X_{0}^{2}+2 Z X_{0} \cos (\theta+\phi)},
$$

and the phase angle $\beta$ can be obtained as

$$
\beta=\operatorname{atan}\left(\frac{\sin (\theta+\phi)}{\cos (\theta+\phi)+\frac{X_{0}}{Z}}\right),
$$

where $\phi=\operatorname{atan}(\Omega / \alpha)$ and the angle $\theta$ can be calculated using Eqs. (21) and (22).

Fig. 6 shows the transmissibility (absolute and relative) and their respective phase angles as a function of the nondimensional frequency. In both results shown in Fig. 6(a) and (b), the red dashed line correspond to unstable trajectory of the frequency response. The loop in absolute transmissibility is due to addition or subtraction of the term $\cos (\theta+\phi)$ described in Eq. (27) as the argument $(\theta+\phi)$ crosses $-\pi / 2$.

\subsection{Determining $X_{1}$ and $Z_{1}$}

Having defined the values of $Z$ and $X$ in the previous Sections, the amplitude of the "internal" displacement $x_{1}$ and $z_{1}=x_{1}-x_{0}$ can be determined using Eq. (2),

$$
X_{1}=\frac{2 \xi_{0} \Omega X}{\sqrt{N^{2}+4 \xi_{0}^{2} \Omega^{2}}}, \quad Z_{1}=\frac{2 \xi_{0} \Omega Z}{\sqrt{N^{2}+4 \xi_{0}^{2} \Omega^{2}}} .
$$

Using the same parameters adopted in Fig. 6, the transmissibilities $X_{1} / X_{0}$ and $Z_{1} / X_{0}$ are plotted in Fig. 7 and compared with $X / X_{0}$ and $Z / X_{0}$.

\section{Stability of the periodic response}

The stability of the periodic response is important in the analysis of nonlinear systems. Loss of stability can be the cause of undesirable jumps in the system response, especially in high frequency. To study the stability of the system, a small perturbation parameter $e$ is used and the response of the system is rewritten as 


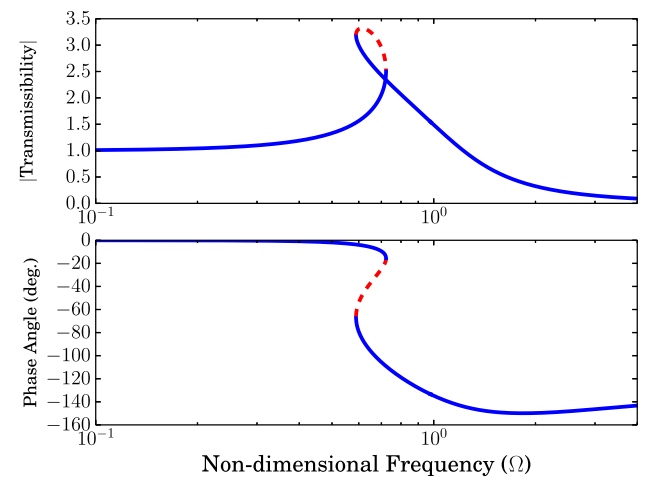

(a)

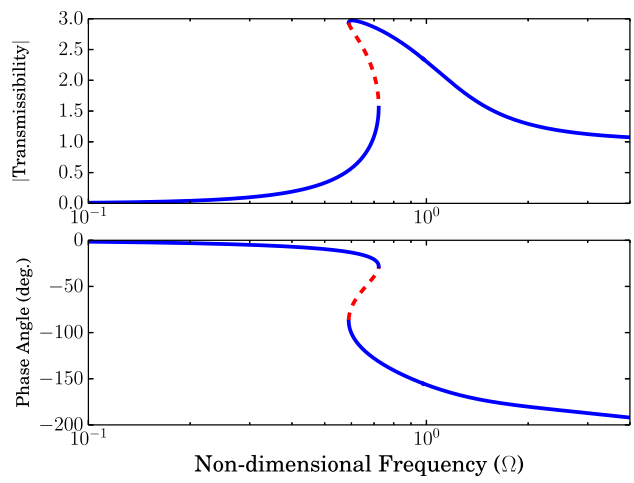

(b)

Fig. 6. Examples of transmissibility magnitude and phase. (a) Absolute transmissibility $\left(X / X_{0}\right)$ and phase angle $(\beta)$, (b) Relative Transmissibility $\left(Z / X_{0}\right)$ and phase angle $(\theta)$. These results where obtained using $\gamma=-0.1, \xi_{0}=0.1, N=3$ and $X_{0}=1$.

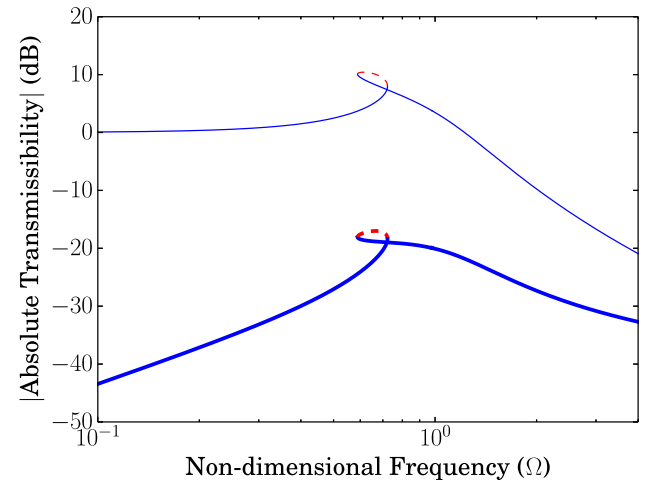

(a)

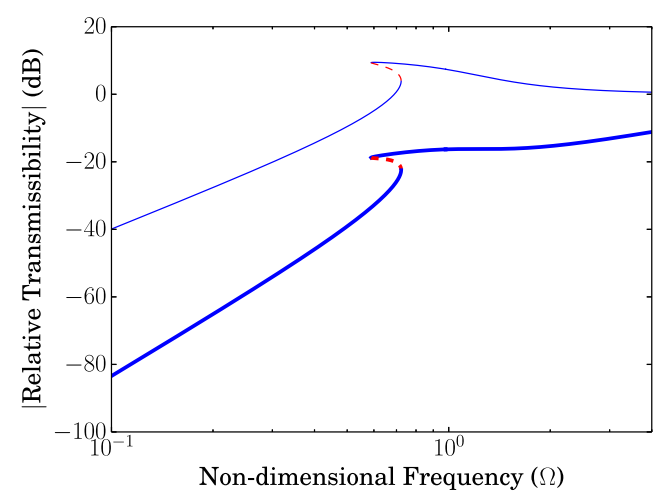

(b)

Fig. 7. Comparison of transmissibility magnitude. (a) Absolute transmissibility $\left(X / X_{0}\right.$ - thin lines) and ( $X_{1} / X_{0}$ thick lines), (b) Relative Transmissibility ( $Z / X_{0}$ - thin lines) and $\left(Z_{1} / X_{0}\right.$ - thick lines). These results where obtained using $\gamma=-0.1, \xi_{0}=0.1, N=3$ and $X_{0}=1$.

$$
z=u(\tau)+e(\tau)=U \cos (\Omega \tau)+e(\tau),
$$

where $u$ is the periodic solution of the equation of motion. Applying Eq. (30) to Eq. (18) and equating to zero gives

$$
\left(u^{\prime \prime \prime}+e^{\prime \prime \prime}\right)+\alpha\left(u^{\prime \prime}+e^{\prime \prime}\right)+\left(N+1+3 \gamma(u+e)^{2}\right)\left(u^{\prime}+e^{\prime}\right)+\alpha\left(1+\gamma(u+e)^{2}\right)(u+e)=0,
$$

since $u$ is a solution of the ODE, the subsequent expression can be equated to zero

$$
u^{\prime \prime \prime}+\alpha u^{\prime \prime}+\left(N+1+3 \gamma u^{2}\right) u^{\prime}+\alpha\left(1+\gamma u^{2}\right) u=0,
$$

leading to a simplification of the solution that enables us to write

$$
e^{\prime \prime \prime}+\alpha e^{\prime \prime}+\left(N+1+3 \gamma(u+e)^{2}\right) e^{\prime}+\alpha\left(1+\gamma(u+e)^{2}\right) e+3 \gamma\left(2 u e+e^{2}\right) u^{\prime}+\alpha \gamma\left(2 u e+e^{2}\right) u=0
$$

As $e$ is a small parameter, the cubic and squared terms $\left(e^{2}, e^{3}\right)$ are neglected, such that

$$
e^{\prime \prime \prime}+\alpha e^{\prime \prime}+(N+1+3 \gamma u(u+2 e)) e^{\prime}+\left(\alpha\left(1+\gamma u^{2}\right)+2 \gamma u\left(\alpha u+3 u^{\prime}\right)\right) e=0 .
$$

At this point, a solution for $e$ is taken to be

$$
e(\tau)=\mathbf{A} \cos (\Omega \tau)+\mathbf{B} \sin (\Omega \tau)
$$

Applying Eq. (35) in Eq. (34), produces two equations as factors of the sine and cosine terms

$$
\begin{aligned}
& \alpha\left(\frac{9}{4} \gamma U^{2}+\left(1-\Omega^{2}\right)\right) \mathbf{A}+\Omega\left(\frac{3}{4} \gamma U^{2}+\left(N+1-\Omega^{2}\right)\right) \mathbf{B}=0, \\
& -\Omega\left(\frac{9}{4} \gamma U^{2}+\left(N+1-\Omega^{2}\right)\right) \mathbf{A}+\alpha\left(\frac{3}{4} \gamma U^{2}+\left(1-\Omega^{2}\right)\right) \mathbf{B}=0 .
\end{aligned}
$$


For nontrivial solutions ( $\mathbf{A} \neq 0$ and $\mathbf{B} \neq 0$ ), the determinant of the matrix defined by Eqs. (36) and (37) must be zero. Taking this determinant,

$$
\left[\frac{27}{16} \gamma^{2}\left(\Omega^{2}+\alpha^{2}\right)\right] U^{4}+3 \gamma\left[\alpha^{2}\left(1-\Omega^{2}\right)+\Omega^{2}\left(N+1-\Omega^{2}\right)\right] U^{2}+\alpha^{2}\left(1-\Omega^{2}\right)^{2}+\Omega^{2}\left(N+1-\Omega^{2}\right)^{2}=0 .
$$

Eq. (38) can be solved in terms of $U$ as a function of $\Omega$, or vice versa. The solution of this implicit equation when plotted in the frequency-amplitude plane, provides the regions of instability (filled red areas) as shown in Fig. 8.

As can be seen from Fig. 8, the boundary of stability regions intersects with frequency response curves where they have vertical slopes, as was expected. These regions are a function of the damping ratio $\xi_{0}$, the stiffness ratio $\gamma$ and $N$ and they do not depend on the base displacement amplitude $X_{0}$.

In this paper, we have concentrated the analysis for the cases were $\gamma<0$ because positive values of $\gamma$ tend to increase the system stiffness with the increase of amplitude. This will cause a degradation of the high frequency response of the isolator when compared with values of $\gamma<0$.

The results shown in Fig. 9 illustrate the boundaries for the regions of instability for $\gamma=-0.1$. These boundaries are defined for the cases of very low damping (blue area) and very high damping (red area). The boundaries defining these areas are defined by the equations shown in this Figure. Increasing the amount of damping, causes the reduction of the area defining the instability as shown in Fig. 10. When damping is too high, there is a change in the region of instability, and increasing damping will cause an increase of size in the region of instability (Fig. 11).

For instance, the results shown in Fig. 12 were calculated for $\xi_{0}=0.1$ and $\gamma=-0.1$ and compared with the linear Zener Model for different values of base displacement amplitude. It can be seen that the resonance and high frequency transmissibility is reduced as $X_{0}$ increases. A drawback is the portion of the frequency response subject to jump phenomena. Similarly, the results shown in Fig. 13 where plotted for $\xi_{0}=1.0$ for various values of base displacement.

As can been seen, the transmissibility responses do not exhibit jumps, however, very large base displacements were necessary to induce the nonlinear response of the system. The high frequency performance is improved without degradation around resonance and at lower frequencies. Similar results shown in Figs. 12 and 13 can be obtained with fixed base amplitude $\left(X_{0}=1\right)$ but increasing the stiffness ratio $\gamma$.

The results shown in Fig. 13 are of particular interest, as they show that adding nonlinear stiffness to the Zener Model with high damping can provide benefits for isolation of vibration. However, to obtain this benefit, it is necessary to have either high values of base displacement or high values of the stiffness ratio.

One important aspect in nonlinear systems with cubic stiffness is the transient response of the system. In many cases, the system may exhibit bistability which depend on the initial conditions. In practice, initial conditions are effectively impossible to control in most real applications, and so all cases must be assumed to be equally likely. However, even solutions on the higher amplitude branch have reduced amplitude compared to the linear case (e.g. blue curves in Fig. 5).

\section{Discussion about optimum damping}

As discussed in the preceding sections of this paper, the transmissibility curve of the traditional Zener Model isolator will vary as a function of the damping value (there exists an optimal damping value as demonstrated in Eq. (8)). This value is obtained noting that all the transmissibility curves pass through the point $\left|T_{c}\right|=(N+2) / N$ which has a corresponding frequency value of $\Omega_{c}=\sqrt{2(N+1) /(N+2)}$ [18,19], regardless of their damping value, as shown in Fig. 14.

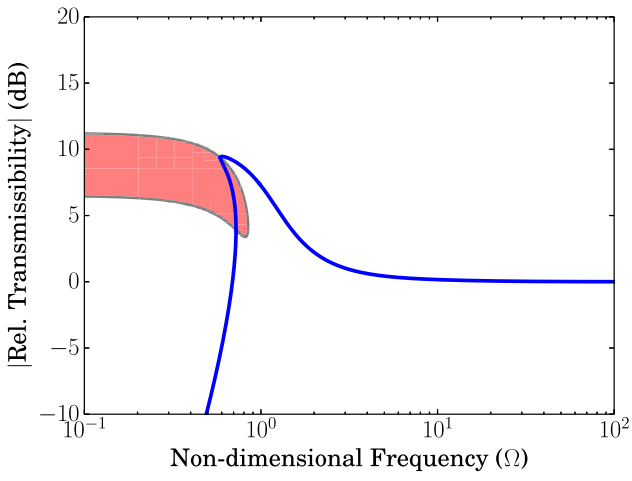

(a)

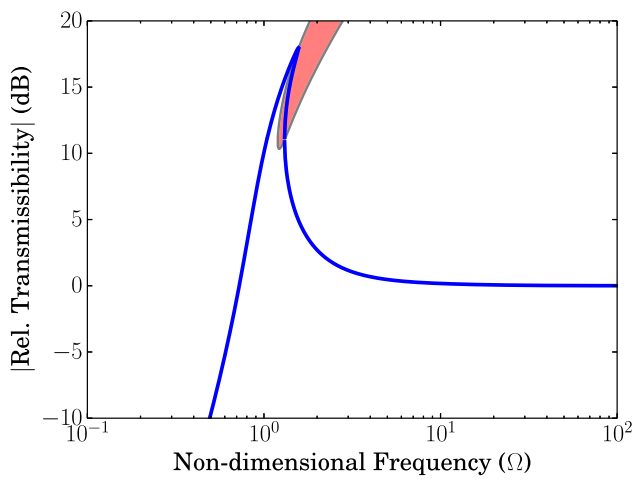

(b)

Fig. 8. Regions of instability defined by the red area. (a) $\gamma<0$ and (b) $\gamma>0$. Parameters used in these results: $N=3, \xi_{0}=0.1, X_{0}=1$. (For interpretation of the references to color in this figure legend, the reader is referred to the web version of this article.) 


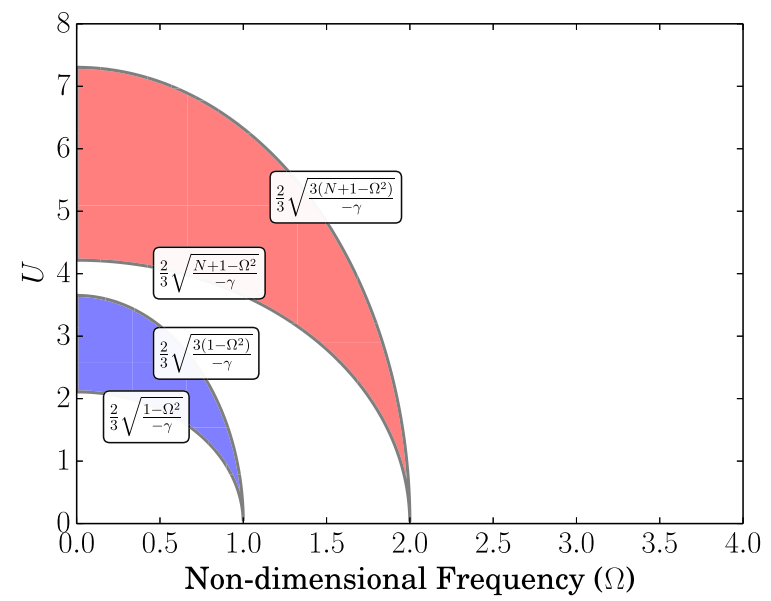

Fig. 9. The regions of instability for low damping (blue area) and high damping (red area), $N=3$. (For interpretation of the references to color in this figure legend, the reader is referred to the web version of this article.)

The optimal damping for a minimum peak is calculated by making the maximum amplitude of the transmissibility occur at frequency $\Omega_{c}$, which is achieved by differentiating the magnitude of the transmissibility with respect to the frequency, evaluated at $\Omega=\Omega_{c}$, such that the slope at this frequency is zero;

$$
\left.\frac{d|T|}{d \Omega}\right|_{\Omega=\Omega_{c}}=0 .
$$

When considering the case of nonlinear stiffness, with negative stiffness ratio $(\gamma)$, a similar behaviour is observed, which can be seen in the results shown in Fig. 15, in which the value of damping ratio $\xi_{0}$ was varied from $1 \times 10^{-3}$ to 100 .

All the curves pass through a value of amplitude and frequency (marked by red dot in Fig. 15), regardless of their damping value. The amplitude of the red dot is defined as

$$
Z_{c}=\frac{N-\sqrt{N^{2}-6 \gamma X_{0}(N-2)}}{3 \gamma X_{0}},
$$

and the frequency is defined as

$$
\Omega_{c}=\frac{1}{2}\left(\frac{2 N\left(3 X_{0}^{2} \gamma+2 N\right) \sqrt{N^{2}-6 X_{0}^{2} \gamma(N+2)}+6 N(N+4) X_{0}^{2} \gamma-4 N^{3}}{3 X_{0}^{2} \gamma \sqrt{N^{2}-6 X_{0}^{2} \gamma(N+2)}-3 X_{0}^{2} \gamma\left(3 X_{0}^{2} \gamma+N\right)}\right)^{1 / 2} .
$$

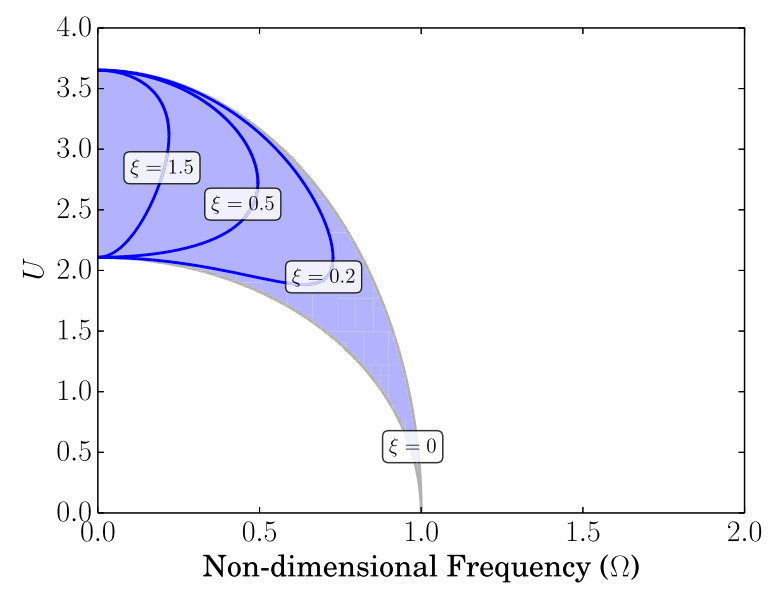

Fig. 10. Regions of instability for low value of damping ratio $\xi_{0}$ with $\gamma=-0.1, N=3$. 


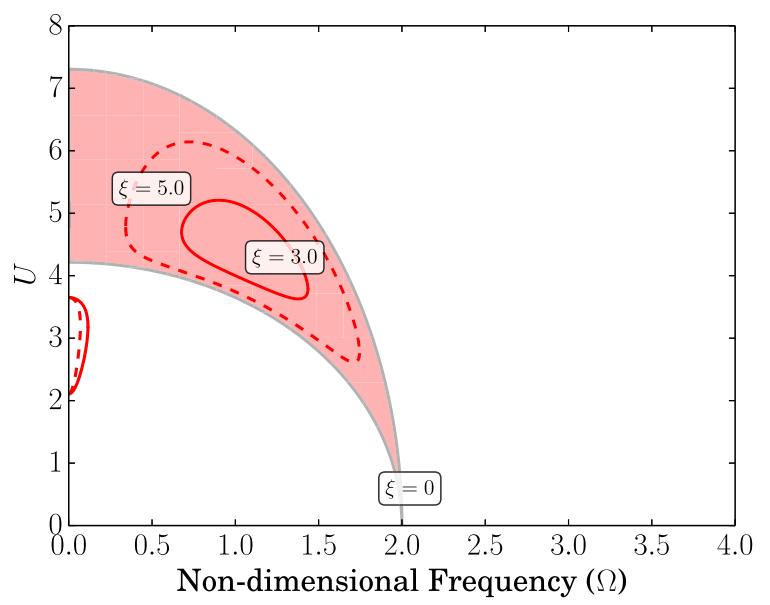

Fig. 11. Regions of instability for high value of damping ratio $\xi_{0}$ with $\gamma=-0.1, N=3$.

Observe that the curves pass through a point that depends on the excitation amplitude $X_{0}$ and the stiffness ratios $\gamma$ and $N$. The transmissibility curve for the nonlinear damping is calculated considering the roots of Eq. (24), so it is necessary to define which root relates to the frequency $\Omega_{c}$.

The hysteresis region which comprises the values of frequency in which the frequency response may assume different values of amplitude can be associated with the discriminant of the polynomial equation

$$
a \bar{Z}^{3}+b \bar{Z}^{2}+c \bar{Z}+d=0
$$

where $\bar{Z}=Z^{2}$ and the discriminant is given by

$$
\Delta=18 a b c d-4 b^{3} d+b^{2} c^{2}-4 a c^{3}-27 a^{2} d^{2},
$$

when $\Delta<0$, Eq. (41) assumes one real and two complex roots. The real root can be computed using the formula described in Ref. [20]

$$
t_{0}=-2 \frac{|q|}{q} \sqrt{-\frac{p}{3}} \cosh \left(\frac{1}{3} \operatorname{arcosh}\left(\frac{-3|q|}{2 p} \sqrt{\frac{-3}{p}}\right)\right), \quad \text { if } 4 p^{3}+27 q^{2}>0 \text { and } p<0
$$

or

$$
t_{0}=-2 \sqrt{\frac{p}{3}} \sinh \left(\frac{1}{3} \operatorname{arsinh}\left(\frac{3 q}{2 p} \sqrt{\frac{3}{p}}\right)\right), \text { if } p>0
$$

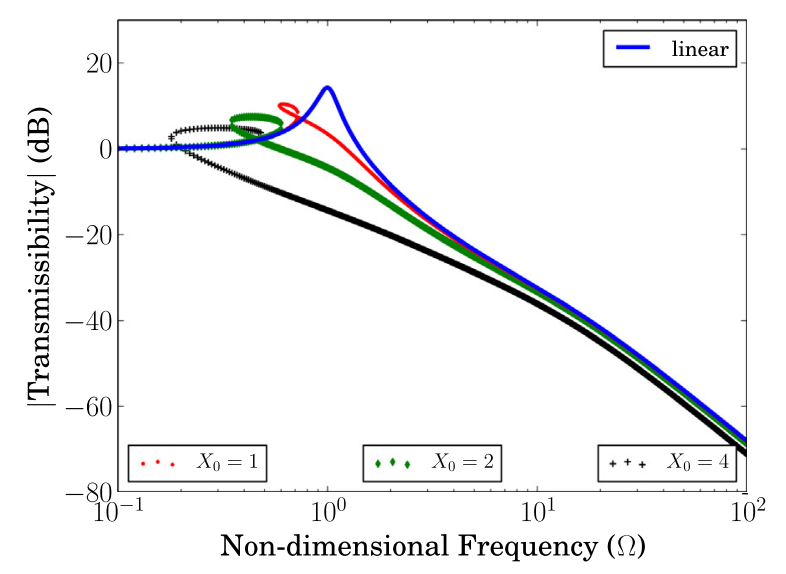

Fig. 12. Comparison of the transmissibility for different values of base displacement, using $\xi=0.1, \gamma=-0.1, N=3$. 


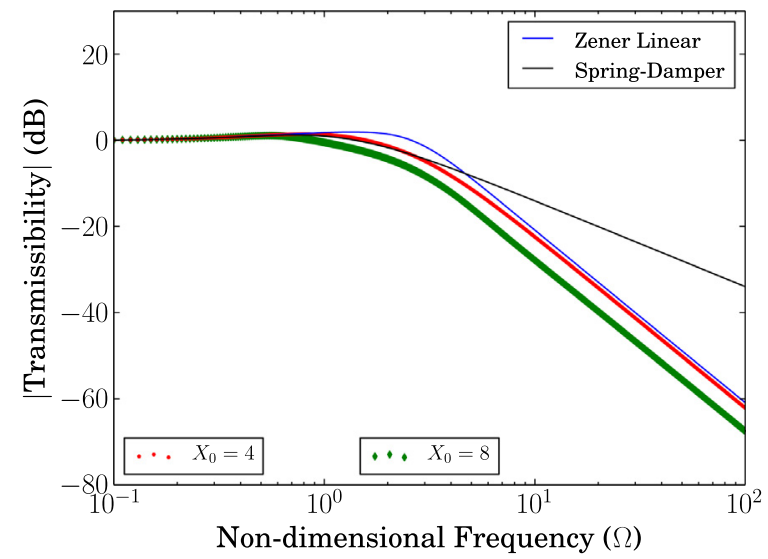

Fig. 13. Isolators transmissibility comparison for different values of base displacement, using $\xi=1.0, \gamma=-0.1, N=8$.

where

$$
\begin{aligned}
& p=\frac{3 a c-b^{2}}{3 a^{2}}, \\
& q=\frac{2 b^{3}-9 a b c+27 a^{2} d}{27 a^{3}} .
\end{aligned}
$$

When $\Delta>0$, Eq. (41) assumes three real roots, and the response can exhibit three possible amplitudes for a given value of frequency, in which two are stable and one is unstable. This is the case of the frequency region where the system may include jumps in the periodic response. In this case, the three roots can be computed using the following equation

$$
t_{n}=2 \sqrt{-\frac{p}{3}} \cos \left(\frac{1}{3} \arccos \left(\frac{3 q}{2 p} \sqrt{\frac{-3}{p}}\right)-\frac{2 \pi n}{3}\right) \text { for } n=0,1,2 .
$$

In any of these cases, the relative displacement is computed as

$$
Z_{n}=\sqrt{\left(t_{n}-\frac{b}{3 a}\right)}
$$

The value of damping for minimum peak transmissibility is calculated assuming that the system response will not include jumps, therefore, according to what was described above in this section, the discriminant of the cubic equation must be negative $(\Delta<0)$. This can be observed in the results shown in Fig. 16(a) where the transmissibility was calculated for different values of damping ratio. The red lines on the bottom of these figures comprises the frequency ranges where the system has

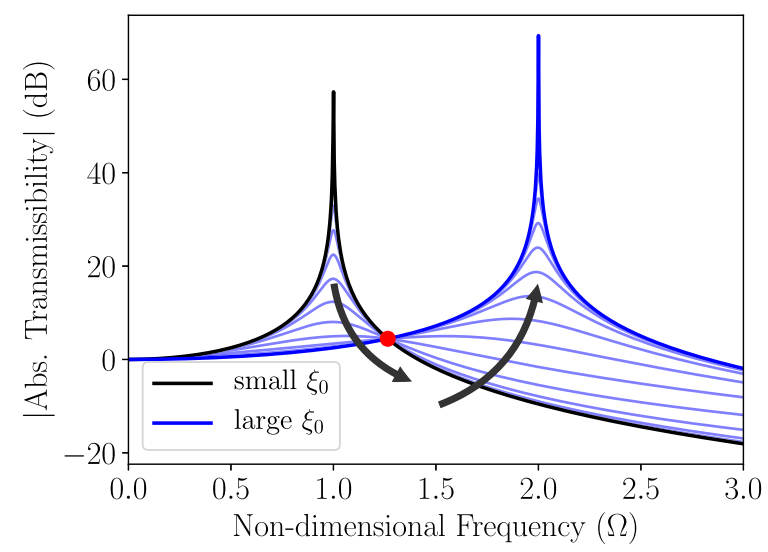

Fig. 14. The absolute transmissibility of the linear Zener isolator for different values of damping ratio. The arrows indicate the direction of increasing the damping ratio. All the curves passes through the red dot defined by the amplitude $\left|T_{c}\right|$ and frequency $\Omega_{c}, N=3$. (For interpretation of the references to color in this figure legend, the reader is referred to the web version of this article.) 


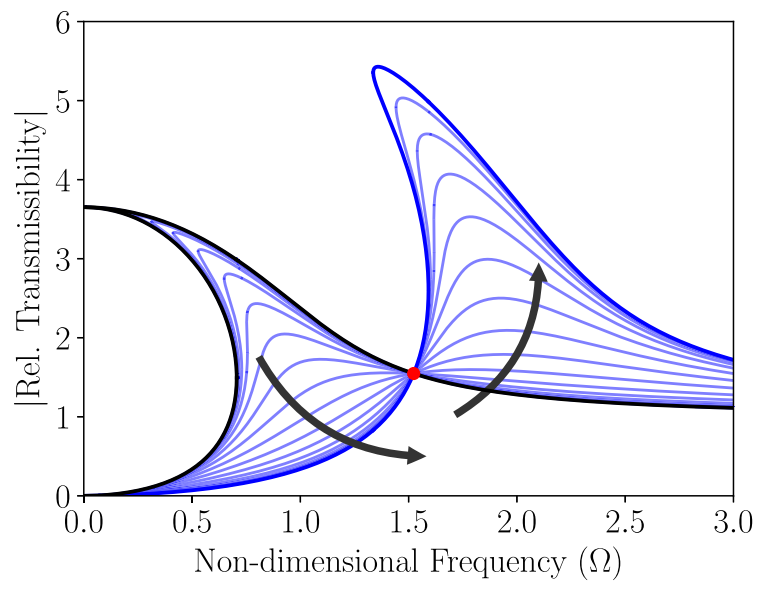

Fig. 15. Relative transmissibility for various values of damping ratio where the arrows indicate the direction of increasing the damping ratio $\xi_{0}$. In these results: $X_{0}=1, N=3, \gamma=-0.1$.

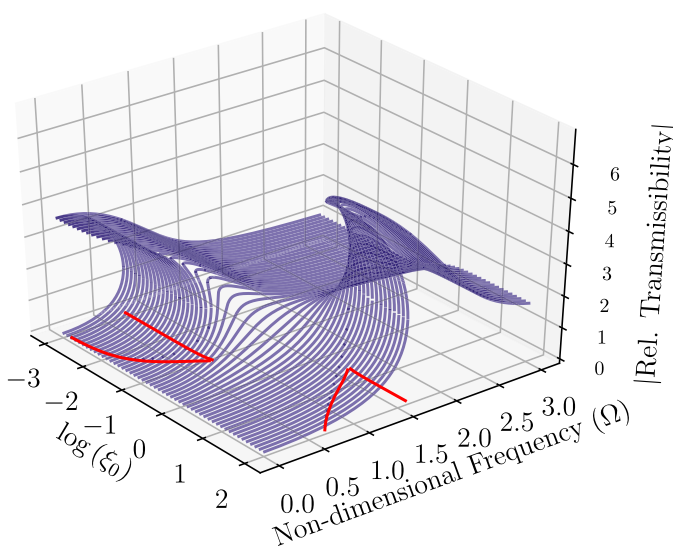

(a)

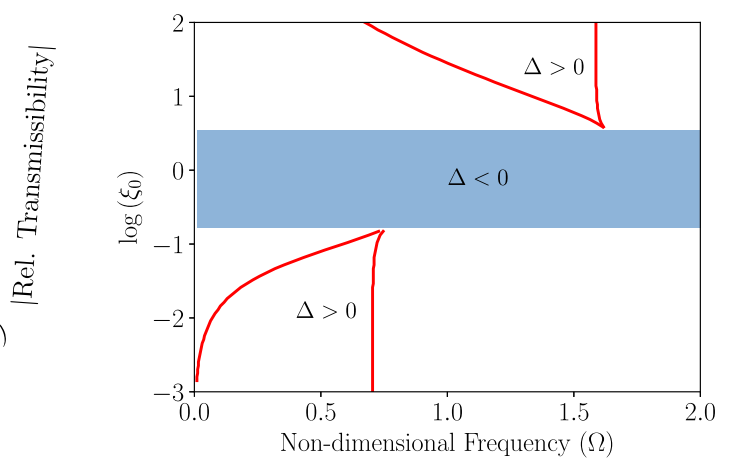

(b)

Fig. 16. (a) Relative transmissibility as a function of the damping ratio. (b) Regions defining the cubic equation discriminant sign.

resonance peaks with positive discriminant. There is a range of damping ratio where the discriminant is always negative, despite the value of frequency, as seen in the detail shown in Fig. 16(b). Considering only the frequency range where $\Delta<0$, the cubic equation will have one single real root, which can be calculated using Eq. (43).

The optimal damping for minimum peak transmissibility is calculated defining a quadratic cost function

$$
\left.J\left(\xi_{0}\right)\right|_{\Omega=\Omega_{c}}=\left.\left(\frac{d Z_{0}}{d \Omega}\right)^{2}\right|_{\Omega=\Omega_{c}}
$$

where the cost function needs to be evaluated only at frequency $\Omega=\Omega_{c} . Z_{c}$ is defined by Eq. (39) and $Z_{0}$ is calculated using Eqs. (43) and (47). In fact, it is very difficult to obtain an exact analytical solution for the optimum damping, however it can be calculated by numerical methods. One way is defined by the following optimization problem

$$
\begin{array}{ll}
\underset{\xi_{0}}{\operatorname{minimize}} & J\left(\xi_{0}\right), \\
\text { subject to } & \Delta\left(\xi_{0}\right)<0, \quad p\left(\xi_{0}\right)<0 .
\end{array}
$$

The cost function $J\left(\xi_{0}\right)$ is defined in the Appendix B. To illustrate the application of this methodology, the results shown in Fig. 17 show the relative displacement for the optimum damping compared with data computed using two different methods. Selecting $N=3, \gamma=-0.1$ and $X_{0}=1$, the value of optimum damping found in the optimization problem was $\xi_{0 o p t}=0.523$. 


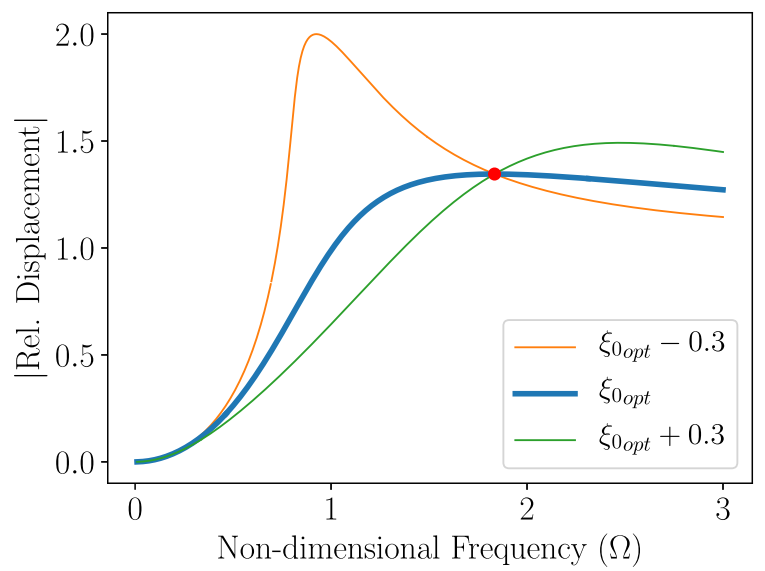

Fig. 17. The relative displacement for different values of damping ratio with $\xi_{0 o p t}=0.523 . N=3, \gamma=-0.1$ and $X_{0}=1$.

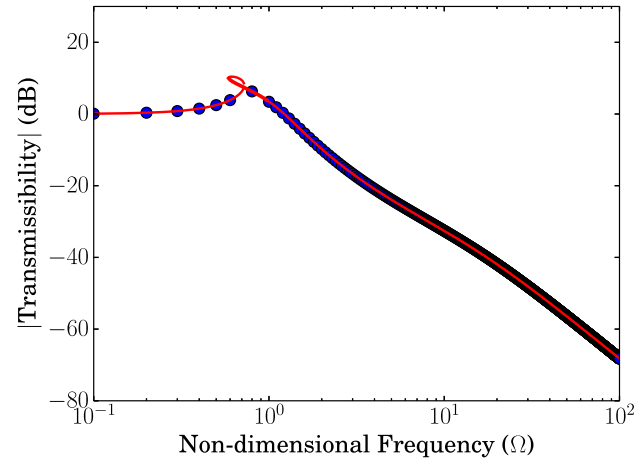

(a)

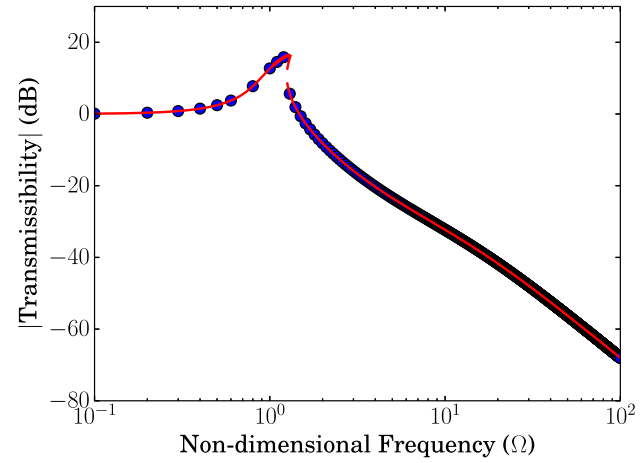

(b)

Fig. 18. Comparison between the absolute transmissibility obtained by numerical integration (dots) and the harmonic balance method (solid line). (a) $\gamma=-0.1$ and (b) $\gamma=0.01$.

\section{Numerical simulations}

To verify the formulation presented in the previous sections, the results obtained using the harmonic balance method were compared with results obtained using numerical integration. For the numerical integration, a 4th and 5th order Runge-Kutta algorithm with a model implemented in the C programming language using the GSL, where the GNU Scientific Library was used. The system was integrated over $600 \mathrm{~s}$ and only the last $10 \%$ of data points were used to compute the maximum amplitude of motion to avoid influence of the transient regime. The analysis was performed for a frequency range of (0.1-100)/ $\omega_{0}$ using $N=3$ and two values of $\gamma$ as shown in Fig. 18(a) and (b). It can be seen that results agree very well, except in the regions of where the response is not stable, where jump phenomena can occur.

The good agreement between the results obtained by numerical integration and the results obtained by first order harmonic balance method is an indication that sub and super harmonics usually present in this type of nonlinearity may be neglected. Also, in the context of this paper, when considering optimal damping, the amplitude of sub/super harmonics tend to be negligible.

\section{Conclusions and final remarks}

This paper has described the dynamic behavior of the Zener model subject to base excitation with the inclusion of a nonlinear stiffness element to improve the system transmissibility at high frequencies. The system was initially described with two degrees of freedom and two equations of motion. Then, these equations where combined in a way that the system was represented by a third order ordinary differential equation. This representation allowed us to write the equations of motion including a nonlinear stiffness element so that the frequency response for the system could then be obtained using the harmonic balance method. 
The main conclusion of the paper is that by including a nonlinear stiffness element in the Zener system it is possible to improve the high frequency transmissibility without degrading the performance across the rest of the frequency range. This is a significant potential benefit for a range of vibration isolation applications. Furthermore, it has been shown how to select the required damping coefficient for the improved isolator system. In addition, it was demonstrated that increasing the values of damping coefficient can be used help to avoid the jump phenomena due to the nonlinear softening stiffness. However, caution is required when designing this feature, as too much damping will cause the system to oscillate at another resonance frequency given by $\Omega=\sqrt{N+1}$.

\section{Acknowledgements}

The authors would like to thank CAPES - Brazil - "Coordenadoria de Aperfeiçoamento de Pessoal de Nível Superior” for providing funding for this research.

\section{Appendix A. Definition of polynomial terms used in the article}

$$
\begin{aligned}
& a=\left(\alpha^{2}+\Omega^{2}\right)\left(\frac{3}{4} \gamma\right)^{2} \\
& b=\frac{3}{2} \gamma\left(\alpha^{2}\left(1-\Omega^{2}\right)+\Omega^{2}\left(N+1-\Omega^{2}\right)\right) \\
& c=\alpha^{2}\left(1-\Omega^{2}\right)^{2}+\Omega^{2}\left(N+1-\Omega^{2}\right)^{2} \\
& d=-X_{0}^{2} \Omega^{4}\left(\alpha^{2}+\Omega^{2}\right) \\
& a_{1}=Z^{2}-X_{0}^{2} \\
& b_{1}=\alpha^{2}\left(Z^{2}-X_{0}^{2}\right)-2 Z\left(N+1+\frac{3}{4} \gamma Z^{2}\right) \\
& c_{1}=Z^{2}\left(N+1+\frac{3}{4} \gamma Z^{2}\right)^{2}-2 \alpha^{2} Z^{2}\left(1+\frac{3}{4} \gamma Z^{2}\right) \\
& d_{1}=\alpha^{2} Z^{2}\left(1+\frac{3}{4} \gamma Z^{2}\right)^{2}
\end{aligned}
$$

\section{Appendix B. Definition of terms used in cost function for optimum damping}

$$
\begin{aligned}
& J\left(\xi_{0}\right)=\frac{-\left(\lambda_{1} \sinh (r)+\lambda_{2}\right)-\lambda_{3} \cosh (r)}{\lambda_{4} \sqrt{-6 a \sqrt{p} \sinh (r)}-\sqrt{3} b} \\
& \lambda_{1}=\sqrt{3} a^{2} p^{\prime} \lambda_{0} \\
& \lambda_{2}=\left(a b^{\prime}-b a^{\prime}\right) \sqrt{p} \lambda_{0} \\
& \lambda_{3}=-3 a^{2}\left(2 p q^{\prime}-3 q p^{\prime}\right) \\
& \lambda_{4}=(48)^{\frac{1}{4}} a^{\frac{3}{2}} \sqrt{p} \lambda_{0} \\
& \lambda_{0}=\sqrt{27 q^{2}+4 p^{3}} \\
& r=\frac{\operatorname{asinh}\left(\frac{3^{3 / 2} q}{2 p^{3 / 2}}\right)}{3}
\end{aligned}
$$


where,

$$
\begin{aligned}
& p^{\prime}=\frac{3 a^{2} c^{\prime}-2 a b b^{\prime}+\left(2 b^{2}-3 a c\right) a^{\prime}}{3 a^{3}} \\
& q^{\prime}=\frac{9 a^{3} d^{\prime}-3 a^{2} b c^{\prime}+\left(2 a b^{2}-3 a^{2} c\right) b^{\prime}+\left(-9 a^{2} d+6 a b c-2 b^{3}\right) a^{\prime}}{9 a^{4}} \\
& a^{\prime}=\frac{9}{8} \gamma^{2} \xi_{0}^{2} \Omega_{c} \\
& b^{\prime}=-\frac{3}{4} \gamma N^{2} \Omega_{c}+3 \gamma \xi_{0}^{2} \Omega_{c}\left(N+1-2 \Omega_{c}^{2}\right) \\
& c^{\prime}=\Omega_{c}\left[\left(2 \xi_{0}^{2}+\Omega_{c}^{2}-1\right) N^{2}+2 \xi_{0}^{2}\left(2\left(1-2 \Omega_{c}^{2}\right) N+\left(3 \Omega_{c}^{4}-4 \Omega_{c}^{2}+1\right)\right)\right] \\
& d^{\prime}=-\Omega_{c}^{3}\left(N^{2}+6 \xi_{0}^{2} \Omega_{c}^{2}\right) X_{0}^{2}
\end{aligned}
$$

\section{References}

[1] R. Ibrahim, Recent advances in nonlinear passive vibration isolators, J. Sound Vib. 314 (2008) $371-452$.

[2] I. Kovacic, M.J. Brennan, T.P. Waters, A study of a nonlinear vibration isolator with a quasi-zero stiffness characteristic, J. Sound Vib. 315 (2008) $700-$ 711.

[3] A. Carrella, M. Brennan, T. Waters, V. Lopes, Force and displacement transmissibility of a nonlinear isolator with high-static-low-dynamic-stiffness, Int. J. Mech. Sci. 55 (2012) 22-29.

[4] A. Carrella, M. Brennan, T. Waters, K. Shin, On the design of a high-static-low-dynamic stiffness isolator using linear mechanical springs and magnets, J. Sound Vib. 315 (2008) 712-720.

[5] A. Shaw, S. Neild, D. Wagg, Dynamic analysis of high static low dynamic stiffness vibration isolation mounts, J. Sound Vib. 332 (2013) $1437-1455$.

[6] P. Guo, Z. Lang, Z. Peng, Analysis and design of the force and displacement transmissibility of nonlinear viscous damper based vibration isolation systems, Nonlinear Dyn. 67 (2012) 2671-2687.

[7] Z. Peng, G. Meng, Z. Lang, W. Zhang, F. Chu, Study of the effects of cubic nonlinear damping on vibration isolations using harmonic balance method, Int. J. Non-Linear Mech. 47 (2012) 1073-1080.

[8] Z. Lang, X. Jing, S. Billings, G. Tomlinson, Z.K. Peng, Theoretical study of the effects of nonlinear viscous damping on vibration isolation of sdof systems, J. Sound Vib. 323 (2009) 352-365.

[9] B. Tang, M. Brennan, A comparison of the effects of nonlinear damping on the free vibration of a single-degree-of-freedom system, J. Vib. Acoust. 134 (2012) 024501.

[10] Z. Peng, Z. Lang, X. Jing, S. Billings, G. Tomlinson, L. Guo, The transmissibility of vibration isolators with a nonlinear antisymmetric damping characteristic, J. Vib. Acoust. 132 (2010) 014501.

[11] Z. Xiao, X. Jing, L. Cheng, The transmissibility of vibration isolators with cubic nonlinear damping under both force and base excitations, J. Sound Vib. 332 (2013) 1335-1354.

[12] Z. Milovanovic, I. Kovacic, M.J. Brennan, On the displacement transmissibility of a base excited viscously damped nonlinear vibration isolator, J. Vib. Acoust. 131 (2009) 054502.

[13] I. Kovacic, On some performance characteristics of base excited vibration isolation systems with a purely nonlinear restoring force, Int. J. Non-Linear Mech. 65 (2014) 44-52.

[14] C. Ho, Z.-Q. Lang, S.A. Billings, Design of vibration isolators by exploiting the beneficial effects of stiffness and damping nonlinearities, J. Sound Vib. 333 (2014) 2489-2504.

[15] X. Sun, X. Jing, Multi-direction vibration isolation with quasi-zero stiffness by employing geometrical nonlinearity, Mech. Syst. Signal Process. 62 (2015) 149-163.

[16] X. Sun, X. Jing, A nonlinear vibration isolator achieving high-static-low-dynamic stiffness and tunable anti-resonance frequency band, Mech. Syst. Signal Process. 80 (2016) 166-188.

[17] J.E. Ruzicka, T.F. Derby, Influence of damping in vibration isolation, Technical Report, DTIC Document, 1971.

[18] J.E. Ruzicka, Forced vibration in systems with elastically supported dampers, Ph.D. thesis, Massachusetts Institute of Technology, 1957.

[19] M. Brennan, A. Carrella, T. Waters, V. Lopes Jr., On the dynamic behaviour of a mass supported by a parallel combination of a spring and an elastically connected damper, J. Sound Vib. 309 (2008) 823-837.

[20] D. Zwillinger, CRC Standard Mathematical Tables and Formulae, CRC Press, 2002. 